\title{
Based VMWARE on
}

\section{the pattern of virtual laboratory to explore}

\author{
Jian Zheng \\ Computer and information engineering college, \\ Shanghai Second Polytechnic University, \\ Shanghai, China \\ zhengjian@sspu.edu.cn
}

\author{
Liyan Cai \\ College of Food and Science Technology, \\ Shanghai Ocean University, \\ Shanghai, China \\ liyan-cai@outlook.com
}

\begin{abstract}
As the funding of practical education in school teaching gradually become rational, coupled with the increasing application of computers in many majors in colleges and universities, the construction of the virtual laboratory has gradually in the implementation. With the above problems, this passage set an example of our school's cloud computing project and construction of software experience center, explored the construction framework of the virtual laboratory and application implementation, and analyzed the effects and problems at present. Through practice, the development of virtual laboratory was further cleared, virtual laboratory construction broadens thought of practical education, integrated facilities resources and network resources, resources can be fully shared, and equipment utilization was improved.
\end{abstract}

Keywords-Cloud computing; VNWARE; The sharing of resources;

\section{INTRODUCTION}

As the continuously improvement of the teaching infrastructure in the campus, the funding into the practical education in the teaching part is going to be more rational. With the development of computer technology, lots of new technology of resources sharing and energy saving are introduced in the campus, for example, cloud computing ${ }^{[1]}$, cloud saving, cloud desktop and cloud application software are gradually applied. The use of these technologies increases the using rate of equipment and data resource and at the same time they have increased managing efficiency.

In the few years, while computer hardware and software are rapidly updated, the network technology is continuously increasing and our society has stepped in the real highway of information. The use of computer in colleges and universities is also enhanced, many are needed in the practical teaching such as the operating system, network and software in the major of computing, auto control and computer aided design in the major of machinery and electronic, image creative packaging design application in the arts specialty, computer aided teaching application in the basic teaching. With the use of much software, the computers need higher management and more maintenance. In spite of the use of hardware-protection technique in our laboratory, new system cannot be used because the operating system updates fast while protection card updates slowly which leads to the result that new system cannot be used. Each computer has to use several systems which lead the waste of resource. There are conflicts in the installation of that software. All above has brought many difficulties to laboratory manager. Because the construction of laboratories has several stages, services in each lab operate almost independently, the resources cannot be fully used and the utilization is quite low.

Almost every college in our university has its own laboratory; there is a phenomenon of repeated construction of the labs though there is a department responsible for management coordination. Since the building for teaching use has reach it limitation, laboratory area is deficient, equipment idle, laboratory is crowded, there is uneven distribution of resources and the resources cannot be shared. At present, the application of computer in teaching is large, there is a large demand of students, and there are nearly 2500 computers in the whole campus with 12,000 students who need to use it, so it brings many pressures to laboratory managers.

To deal with the demand for students' using computers, the main idea is to make use of their own resources, the virtual technology and the cloud technologies, students can feel convenience to use the data by the usage of cloud desktop.

In 2013 and 2014, the college of computer and information engineering began the first construction of the cloud computing combined with the construction of software experience center and application of cloud desktop at the same time, through the operation in the first phase, we have been programming the second phase of according to the existing problems.

\section{STUDY ON THE MODEL OF VIRTUAL LABORARORY BASED ON CLOUD COMPUTING}

\section{A. The concept of cloud computing}

Cloud computing is mainly based on the pattern of increase, use and delivery of related services on the internet which can provide dynamic, easy-to-extend and virtual resources through the internet. Its widely acceptable definition is come from the U.S. national institute of standards and technology (NIST) ${ }^{[2]}$ : cloud computing is a pattern of payment according to the usage, this pattern provides convenient, on-demand network view and 
available access into the configurable computing resources sharing pool (resources including network, servers, storage, applications, services), these resources can be quickly provided which need few managements or little interaction with service providers.

Currently, cloud computing has the ability to operate 10 trillion times a second; it can simulate nuclear explosion, predict climate change and market development trend. Now many users have access to the data center through terminal equipment like laptop and mobile phone to get the resources that they demand.

\section{B. Accomplishment of virtual laboratory pattern}

In the first construction period, we introduce the cloud computing and cloud management desktop combined with the construction of software experience center in a pilot project.

The basic framework is shown in picture 1, it is consisted of two parts: one is cloud computing center room, its main equipment is ten blade servers and storage which is responsible for cloud computing, cloud service and application, the other is software experience center laboratory, its main equipment are two interchangers, two servers and a storage which is responsible for cloud management, cloud service and application, this dual-server backup is used for safety operation.

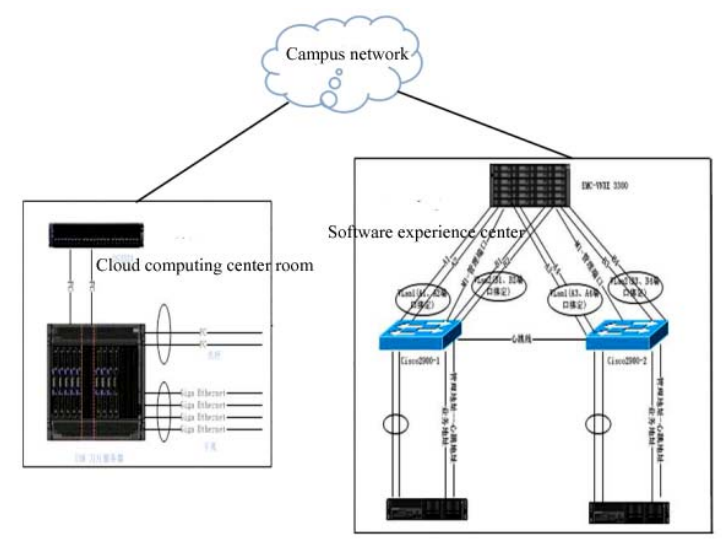

Pig. 1 Cloud computing system framework

The entire cloud system in the campus network is in the same domain controller, all the resources are put into a domain, managed by a VMware Virtual Center management system, it provides a scalable, expansible platform, which laid a foundation for virtualization management. VMware Virtual Center can centrally manage VMware Sphere environment, compared with other management platform it can greatly improve the network administrators' control of the Virtual environment. VMware Virtual Center is a 64 - bit Windows application which drastically improves the scalability. A VMware Virtual Center can manage up to 1000 host computers and 10,000 running Virtual machine, under the link mode, you can manage up to 30000 Virtual machine through 10 VMware Virtual Center. VMware HA instance management and VMware DRS cluster can support up to 32 host computers and 3000 Virtual machines.
In the first phase, VMware Virtual Center can do the following management:

(1) Users management

(2) Cloud desktop management

(3) Virtual service management (documents, e-mails, safety)

(4) Management of all business

Through configuration, it provides all teachers with cloud desktop; teachers can go on with their teaching activities such as preparing lessons, operation demonstration on cloud desktop. We provide remote dial-in service in the virtual service ${ }^{[3]}$, so it's convenient for teachers use cloud desktop outside school. At the same time, we provide cloud desktop service for students in college of software engineering. We install cloud desktop on the computers in software experience center which can let students log in their own desktop easily and use some large database software to excise. Cloud a desktop support Windows ISO Linux desktop platform which is convenient for us to carry out various operations and learning in the operating system and develop and practice corresponding software running on this platform.

\section{THE VIRTUAL LABORATORY CONSTRUCTION}

By using related system and resources of the school through VPN ${ }^{[4]}$, teachers can easily prepare for their courses in the cloud desktop, demonstrate related operations of the course and can simulate the environment of computer labs by themselves. Students can use cloud desktop to operate various software, they can install the software that cannot be installed in their own computer, but it's available in the cloud desktop, such as Linux, OS2 and so on, as long as the students install a login-on program, you can open a virtual system in your computer and do any computer experiments through a permissions set. In the cloud server, there is mail service, online security protection software being installed, visitors can take advantage of these services to protect and easily use cloud desktop and their local computer. The dynamic migration ${ }^{[5]}$ of Cloud computing in the cloud server is more convenient and quick than entity machines; it's helpful for the adjustment of system resources. By the establishment of virtual laboratory, the school resources are unified managed, resources are shared which greatly improve the security of the computer and achieve desired effect.

\section{The Probllem of Virtual LabPratory at Present}

Through the construction of the virtual laboratory we found some problems, for example, the cloud computing management need a special system manager to do the management and maintenance, high technical requirements of laboratory management and it's a lasting work which has a great potential in the updates, application and development of technology and which demand for unceasingly exploration. At present, some teachers are still not familiar with computer application and feel tedious with the operation. Because the cloud computing services frequently use online resources, there is a higher requirement on network speed. The network speed in the campus is quite high while the network speed at home is slow, 
so there is a lower speed when using the cloud desktop. As we can see, the emergence of a new model will often come to the situation of many problems; virtual laboratory ${ }^{[6]}$ can develop well in the future which is now in gradually promotion. We will further increase investment in manpower and material resources in this aspect, fully embodies the advantages of virtual laboratory.

\section{CONCLUSION}

This paper studies the construction of virtual process laboratory based on VMWARE, give an example of the school cloud computing experience center construction project and instance of software construction project. It proves that the construction of virtual laboratory plays an irreplaceable role in practical teaching. Striving to develop the construction of virtual laboratory and further optimize the construction has a profound influence on current development of college practical teaching. Finally, thanks to the funding of the key disciplinary subject computer application technology (cultivating) (No. XXKPY1301).

\section{REFERENCES}

[1] LI An, ZHOU Nan-run, WANG Yu-hao. Construction and Practice of the Construction of a National Virtual Lab for Communication Engineering [J]. RESEARCH AND EXPLORATION IN LABORATORY. 2013(01):150-154.

[2] Gao Lin, Song Xiang-Qian, Wang Jie-Ping. Research on cloud computing and key technologies [J]. Microcomputer \& Its Applications.2011(10):5-7.

[3] WANG Tai-cheng, CAI Yong. Using virtual Machine Technology to Perform Complex Network Experiment. [J].COMPUTER TECHNOLOGY AND DEVELOPM ENT.2009(04):246-249.

[4] CHEN Guo-wei. Study on application of virtual machine technology in practical teaching of network technology [J]. Experimental Technology and Management.2014(05):115-117.

[5] JIAO Shu-jin. In cloud computing technologies of the virtual machine live migration [M]. Taiyuan University of Technology.2013.5.1.

[6] Wu Xin-hua Wang Li-ping. Design and Implementation of the Virtual Computer Laboratory Based on Cloud Computing [J]. Journal of Pingxiang College.2014(06):60-62. 